\title{
Conformation and Energy Transfer in a Single Luminescent Conjugated Polymer
}

\author{
Wen-Yu Sun, ${ }^{\dagger, \ddagger}$ Shu-Chun Yang, ${ }^{\dagger, \$}$ J. D. White, ${ }^{*, \dagger, \|}$ Jui-Hung Hsu, ${ }^{\perp}$ K.-Y. Peng,, \\ S. A. Chen, ${ }^{\#}$ and Wunshain Fann $*,+, *$ \\ Institute of Atomic \& Molecular Sciences, Academia Sinica, Taipei, Taiwan; Department of Physics \\ and Institute of Polymer Science and Engineering, National Taiwan University, Taipei, Taiwan; \\ Department of Physics, Tamkang University, Tamshui, Taiwan; Department of Electrical Engineering, \\ Yuan Ze University, Taoyuan, Taiwan; Department of Materials and Optoelectronics Engineering, \\ National Sun Yat-sen University, Kaohsiung, Taiwan; and Department of Chemical Engineering, \\ National Tsing Hua University, Hsinchu, Taiwan
}

Received June 30, 2004; Revised Manuscript Received December 7, 2004

\begin{abstract}
The photophysics and conformation of single long-chain 2,5-dioctyloxy-p-phenylenevinylene (DOO-PPV) polymers are investigated. The fluorescence intensity-time trace of an individual polymer contains abrupt quantized intensity changes superimposed on small gradual changes. Under the same processing conditions, the size of abrupt changes varies from individual to individual, varying between 0 and $100 \%$ of the total intensity. Polarization modulation indicates considerable orientation of absorption dipoles within the polymer. Time-dependent measurements indicate that, in the majority of polymers, absorption dipoles in those regions of the polymer responsible for abrupt intensity changes are arranged less anisotropically than in regions responsible for the gradual changes. Spectroscopic measurements show a greater spectral variation accompanying jumps than that accompanying gradual decay. This can be explained by the coexistence of extended regions and a core region. The first is characterized by a relatively greater alignment of absorption dipoles and multiple emitters, while in the second, absorption dipoles are more isotropically distributed and energy is efficiently funneled to a few emitting excitons. Within a single processing batch, the ratio of these two regions varies from individual to individual.
\end{abstract}

\section{Introduction}

Luminescent conjugated polymers have been the focus of extensive research due to their potential application as the active layer in a flat panel display ${ }^{1}$ and interesting photophysical properties. Considerable effort has been expended in trying to understand the relationships between energy transfer, quenching, and conformation and their effects on the photophysics in the condensed phase. Single molecule spectroscopy (SMS) has shown that the behavior of a single polymer is different from the on-off blinking observed in the fluorescence of small molecules. ${ }^{2}$ Within the family of PPV ( $p$-phenylenevinylene) polymers, such single polymer studies can be divided into two categories: the majority that focus on long-chain polymers (primarily MEH-PPV) and a few investigating simpler, persistent length polymers (i.e., poly[2,5-dioctyloxy (DOO)-PPV). For short chain polymers, the fluorescence time trace is characterized by emission at a few discrete intensities, ${ }^{3}$ the number of which corresponds to the number of chromophores or excitons expected theoretically. ${ }^{4}$ Data obtained by rotating the excitation polarization and observing the modulation in fluorescence indicate that conformation is predominately rod-shaped, except for kinks attributed to tetrahedral defects. ${ }^{5}$ In such a system, energy transfer between excitons has been shown to be quite inefficient. ${ }^{6}$ The picture is more complicated for long chain MEH-PPV with the nature of the fluorescence time trace strongly dependent on processing condi-

\footnotetext{
$\dagger$ Academia Sinica.

Taiwan University.

$\S$ Tamkang University.

"Yuan Ze University.

$\perp$ National Sun Yat-sen University

\# National Tsing Hua University.
}

tions. ${ }^{7-12}$ Simulations on the collapse of a polymer found tetrahedral defect density to be a key parameter in determining the final conformation of the polymer. ${ }^{7}$ Experimentally, Yu et al. observed emission from MEHPPV at only a few discrete intensities, leading to the conclusion that there is very efficient "energy funneling" from many absorbing excitons to a few, lower energy, emitting segments. ${ }^{8}$ When dissolved in tetrahydrofuran (THF) and deposited by ink jet printing, the fact that fluorescence time trace was similar to small molecules was taken to indicate the existence of only a single emissive site. ${ }^{11}$ While Huser et al. observed similar behavior for MEH-PPV dissolved in toluene, they observed that replacing toluene with chloroform resulted in an emission that decreased gradually with only small discrete jumps occurring later in the time trace. ${ }^{10}$ Their conclusion was that the conformation of MEH-PPV is solvent dependent. Toluene causes MEH-PPV to "selfaggregate" with energy being transferred to a single emitter while in chloroform the polymer remains extended with multiple emitters. ${ }^{9}$ While the above results indicate that MEH-PPV can adopt two distinct types of behavior, a number of questions remain. Do processing conditions dictate the conformation of the individual polymer, or can some individuals within the same batch exhibit the first type of behavior and other individuals the second? Taking this a step farther, within an individual polymer, can some regions of the polymer exhibit the first type of behavior and some regions the second type? Finally, is this type of behavior unique to $\mathrm{MEH}-\mathrm{PPV}$, or is it a general characteristic of the PPV family?

To answer these questions and to obtain a better understanding of the conformation of individual PPV derivative polymers, we chose to make the first single 


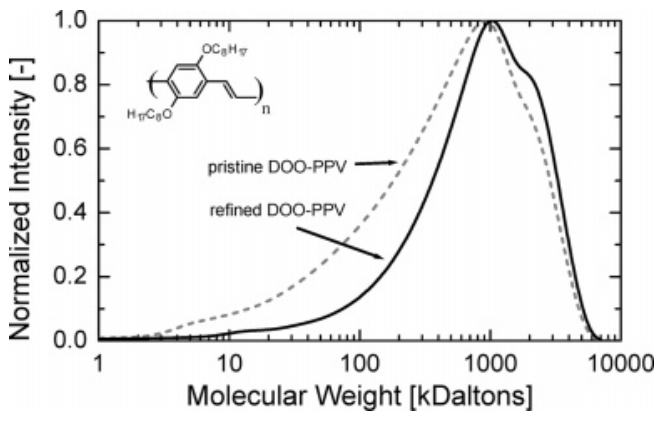

Figure 1. Gel permeation chromatography (GPC) results for pristine and refined DOO-PPV. Calibration was with polystyrene standards. The solvent was chloroform. The inset shows the structure of the DOO-PPV monomer.

molecule investigation into long-chain dioctyloxy-PPV (DOO-PPV). DOO-PPV, in contrast to highly soluble, asymmetrically substituted MEH-PPV, is a symmetrically substituted polymer (see Figure 1(inset) for structure) known for its poor solubility which depends strongly on both solvent and temperature. The various photophysics properties of DOO-PPV, including its cooperative and stimulated emission properties ${ }^{13,14}$ and even-parity states, ${ }^{15}$ as well as possible application in ultrafast excitonic switching ${ }^{16}$ have been investigated. While a molecular exciton based model with one emissive species has been found to adequately explain the absorption and PL spectrum of dilute solutions MEHPPV, ${ }^{18}$ difficulties have been found in employing the same model for DOO-PPV in poor solvent. However, Chang et al..$^{19}$ have shown that both the PL spectra and dynamics of the time-resolved PL can be explained, within the context of the above model, by the coexistence of an intrachain and one kind of aggregate (interchain) state whose quantum efficiency is less than the former state. In addition to solubility and chemical structure induced differences, the DOO-PPV studied in this work has a low tetrahedral defect rate $(<1 \%)$ compared with standard MEH-PPV ( $5 \%)$. Granted the influence of solubility, chemical structure, and $\operatorname{defects}^{7}$ on polymer conformation, it is not clear whether single DOO-PPV will exhibit similar behavior and adopt a similar conformation to MEH-PPV.

\section{Experimental Methods}

DOO-PPV, having $<1 \%$ tetrahedral defects (as determined by NMR using the technique of Becker ${ }^{20}$, was synthesized following a procedure similar to that used by Holmes and coworkers ${ }^{21}$ and Wudl and co-workers. ${ }^{22}$ The molecular weight (weight-average) $\left(M_{\mathrm{w}}\right)$ of the pristine polymer is estimated to be $900 \mathrm{kDa}$ with a polydispersity $(\delta)$ of 8.6 as determined by gel permeation chromatography (GPC) calibrated with polystyrene standards (Figure 1, gray dashed line). While this molecular weight and polydispersity are typical of those used in conjugated polymer experiments, a direct study of this polymer may result in ambiguity in the results due to the influence of shorter chain polymers. Two length scales are of interest here: the first is the persistence length of the polymer $\left(l_{\mathrm{p}}\right)$ and the second the mean distance between defects $\left(l_{\mathrm{d}}\right)$. Polymers that are on the order of a persistence length or that are defect-free would be expected to exhibit quite different conformation than longer chain polymers. (For the above polymer, $12 \%$ of the polymers are shorter than $11 l_{\mathrm{p}}$ and $1.7 l_{\mathrm{d}}$.) To reduce the number of shorter chains in the sample and thus remove ambiguity, we have taken advantage of the limited solubility of this polymer, ${ }^{23}$ immersing the pristine DOO-PPV in $40^{\circ} \mathrm{C}$ chloroform and discarding the dissolved fragments. The molecular weight of the resulting polymer, as estimated by gel permeation chromatography (GPC) using chloroform as the solvent, is $1300 \mathrm{kDa}$ with a polydispersity $(\delta)$ of 4.0 (Figure 1 , solid line). The refinement process has thus reduced the percentage of polymers in the tail having lengths $<11 l_{\mathrm{p}}$ and $<1.7 l_{\mathrm{d}}$ to $<4 \%$ of the sample population. Considering the fact that in this study $\sim 80$ polymers were observed, one expects that only three of these polymers will exhibit behavior characteristic of shorter chain lengths. Further evidence that short chain polymers have been removed is seen in the PL spectra in solution previously published by Hsu et al. ${ }^{23}$ in which the refined spectrum lacks the blue emission contributed by short chain polymers in the pristine sample. (This polymer contains $\sim 3000$ monomer units, and $\sim 500$ chromophores, assuming six monomers per chromophore. $\left.{ }^{4}\right)$

The residual powder was then dissolved in $50^{\circ} \mathrm{C}$ chloroform solution for a day. The dispersed single-molecule stock solution, after further dilution in a polystyrene matrix ( $\sim$ polystyrene:chloroform:toluene ( $5 \mathrm{mg}: 8 \mathrm{~mL}: 2 \mathrm{~mL})$ ), was spin-cast onto a fused silica coverslip to form a film thickness of $<100 \mathrm{~nm}$ and moved into a dry nitrogen glovebox. The film was allowed to dry under low pressure for a number of hours before the nitrogen pressure within the chamber was raised to atmospheric pressure. The sample was then sealed in a specially designed holder to reduce the contact of the film with the ambient environment ${ }^{5}$ as the presence of oxygen sets the time scale for the experiment. Under ambient conditions sample lifetimes under excitation are only a few seconds, and the photons available from a single polymer limits one to an integration time of $\sim 10 \mathrm{~ms}$. Thus, protection is necessary to extend polymer lifetime in order to reduce the probability of multiple quench events occurring within a single time interval. ${ }^{24,25}$ Such events would mask both dark states and energy transfer ${ }^{3,8}$

The protected sample was observed using confocal fluorescence microscopy in standard epi-fluorescence geometry. ${ }^{26}$ After raster scanning the sample, individual polymers were systematically positioned at the focal point of the objective lens (Nikon $100 \times$ oil immersion, N.A. $=1.3$ ), excited by a continuous wave argon laser (488 $\mathrm{nm}$, linearly polarized), and their fluorescent time trace recorded by an avalanche photodiode operating in single photon counting mode.

The alignment of absorption dipoles $(\mu)$ within the polymer was investigated by slowly rotating the polarization of the excitation light at $5 \mathrm{~Hz}$ using an electrooptical modulator $(2.5$ $\mathrm{Hz})$ and observing the changes in emission intensity. ${ }^{27}$ This technique takes advantage of the fact absorption and thus fluorescence are proportional to $|\mu \cdot \mathbf{E}|^{2}$, where $\mathbf{E}$ is electric field vector. If neither the absorption dipoles nor excitation light has a component perpendicular to the sample plane, this provides unambiguous information on the orientation of the absorption dipole. ${ }^{28}$ While not crucial for this experiment, in the prepared sample, the first condition is nearly met as the dipoles are randomly oriented primarily in the sample plane. ${ }^{11}$ The second condition is met by underfilling the objective lens in order to avoid those sections which contribute to a field component perpendicular to the sample plane.

Spectral changes during the fluorescence time decay were investigated by splitting the PL into two channels. The first channel was directed toward an APD in order to monitor the nature of the fluorescence time decay while the second channel was directed into a monochromator/liquid nitrogen-cooled CCD detection system.

\section{Results and Discussion}

Time-Averaged Photoluminescence (PL) Spectrum. Figure 2 compares the PL spectrum of DOO-PPV embedded in polystyrene with that recorded in good and poor solvents. The PL spectrum of DOO-PPV (Figure 2 , dotted line) diluted in chloroform (good solvent) is similar to MEH-PPV and is peaked at $550 \mathrm{~nm}$ and has shoulder at 595 with a relative height of 0.6 . Generally, the Huang-Rhys factor $(S)$ of $\leq 0.6$ for conjugated polymers indicates a single emissive state which has been attributed to intrachain emission. ${ }^{17}$ Good agree- 


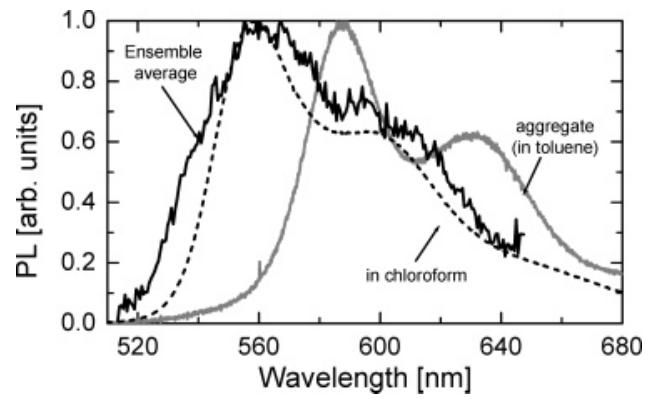

Figure 2. Photoluminescence (PL) spectra of long-chain DOOPPV polymers excited at $488 \mathrm{~nm}$. The dashed black line is the spectra recorded for the polymer diluted in chloroform (good solvent). The gray line is the difference between the spectrum in toluene (poor solvent) and that in a good solvent, in other words, the spectrum of an aggregate. ${ }^{17}$ The solid black line is an ensemble average of the spectra of single polymers embedded in the polymer matrix. This spectrum cuts off at $650 \mathrm{~nm}$ due to the limited bandwidth of the monochromator/CCD combination used in our experiment $(140 \mathrm{~nm})$.

ment has been found between this spectra and the predictions of a molecular exciton based model. ${ }^{18,19}$ The PL spectra of highly dilute DOO-PPV in toluene (poor solvent) can be decomposed into two components: an intrachain component (similar to that in chloroform) and an "aggregate" component (Figure 2, gray line) ${ }^{19}$ having a peak at $595 \mathrm{~nm}$ and a shoulder at $630 \mathrm{~nm}$. The second is similar to that of DOO-PPV deposited in a thin film. Its Huang-Rhys factor is also 0.6, indicating that this spectrum also represents a single emissive species. Above $50{ }^{\circ} \mathrm{C}$ the spectrum in toluene is characterized solely by an intrachain component, but as temperature drops below room temperature, the "aggregate" component increasingly dominates. The ensemble single polymer spectrum under our deposition conditions (Figure 2 , solid line) has a peak at $\sim 560 \mathrm{~nm}$ and has a shoulder at $\sim 590 \mathrm{~nm}$ having a relative height of $\sim 0.75$. This additional height at the shoulder suggests that polymers investigated in this experiment may have two states contributing to emission. The actual emission spectrum of a single polymer both varies from individual to individual and between different periods in the polymer's lifetime. This variation is, however, much less than in short chain DOO-PPV, where the spectrum of an individual polymer is considerably narrower than the ensemble average, and the peak position varies from individual to individual. ${ }^{3}$

Fluorescence Time Trace. The fluorescence time trace provides valuable information on energy transfer and the number of emitters in a polymer. A trace dominated by one or two large steps would indicate either very few absorbing and emitting sites or very efficient energy transfer from many absorbing sites within the polymer to a few emitting sites. Decay dominated by many small steps would indicate that there exist many absorbing and emitting chromophores. Figures 3-5 present fluorescence time traces representative of the behaviors observed in the sample. For the polymer in Figure 3, the fluorescence decays smoothly during the first $12 \mathrm{~s}$, with one distinct drop occurring at $\sim 5.8 \mathrm{~s}$, which recovers $\sim 0.4 \mathrm{~s}$ later (see inset). From 12 to $25 \mathrm{~s}$ emission is relatively constant, at which point the intensity drops quickly, but not in a single step (on the $10 \mathrm{~ms}$ time scale of the experiment), to about half its previous value. At $28 \mathrm{~s}$ the emission drops abruptly to near background level. Emission remains near background until $76 \mathrm{~s}$ when an abrupt jump in intensity

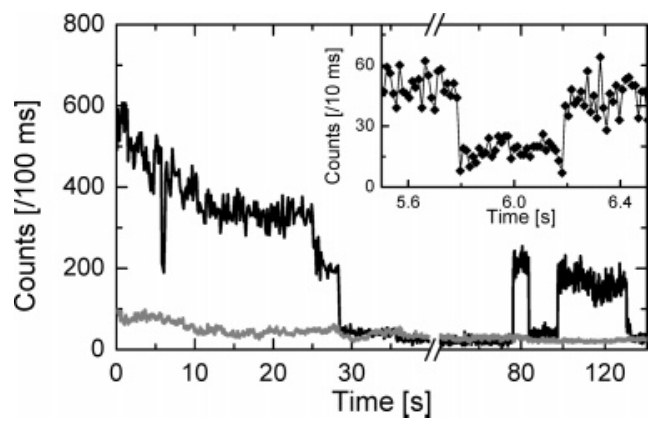

Figure 3. First $140 \mathrm{~s}$ of the fluorescence time trace of single long-chain DOO-PPV polymer under excitation with linearly polarized light. Excitation irradiance is $\sim 1600 \mathrm{~W} / \mathrm{cm}^{2}$, and the background level is $\sim 4$ counts $/ 10 \mathrm{~ms}$. The PL from the polymer is denoted by the solid black line while the background (taken at a point where there is no polymer) is denoted by the light gray line. Note that there is a break in scale between 30 and $60 \mathrm{~s}$ as during which time there is no fluorescence from the polymer. While in the main figure, the bin size is $100 \mathrm{~ms}$, the inset expands the area around $6 \mathrm{~s}$ to show that the drop in intensity is real and occurs within the $10 \mathrm{~ms}$ time scale of this experiment. (It should be noted that the count rate is low due to the use of a low efficiency detector (this figure only).)

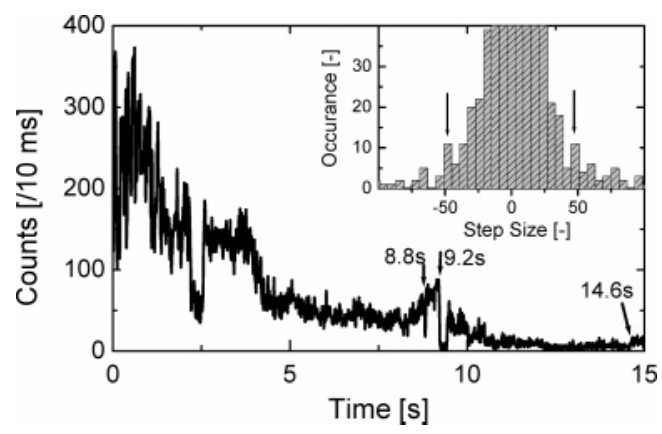

Figure 4. First $15 \mathrm{~s}$ of the fluorescence time trace of single long-chain DOO-PPV polymer under excitation with linearly polarized light recorded with $10 \mathrm{~ms}$ time resolution. Excitation irradiance is $1600 \mathrm{~W} / \mathrm{cm}^{2}$. In contrast to the previous polymer, at short time intervals this polymer exhibits multistep emission while at a longer time scale there is a slow smooth decay in emission intensity. The inset is a histogram of the change in intensity between each time interval. While the changes in intensity are dominated by Gaussian noise, the two strong peaks (marked by arrows) at \pm 48 counts $/ 10 \mathrm{~ms}$ are from repeated abrupt intensity changes.

occurs. Such on-off behavior occurs out to $170 \mathrm{~s}$ (when we stopped recording data, although only the first 140 $\mathrm{s}$ of the molecule lifetime is shown). For this molecule, abrupt changes in intensity (drops or jumps) occur at $5.8,6.2,28.4,76.2,83.7,97.4,130.5 \mathrm{~s}$, and in each case the change of intensity $(\Delta)$ is the same $(\Delta \sim 19 \pm 5$ counts $/ 10 \mathrm{~ms}$ ). Other changes in intensity are gradual and less than shot noise.

For the polymer in Figure 4, the first $3 \mathrm{~s}$ is characterized by both large abrupt fluctuations in emission and smaller changes in intensity on the order of the shot noise. No abrupt changes of intensity occur between 3 and $8.8 \mathrm{~s}$. At $8.8 \mathrm{~s}$ there is an abrupt drop $(\Delta \sim 48$ counts/10 ms) and asequent jump in intensity by the same amount $100 \mathrm{~ms}$ later. At $9.2 \mathrm{~s}$ there is another abrupt drop in intensity ( $\Delta \sim 70$ counts $/ 10 \mathrm{~ms}$ ) followed by a jump in intensity $200 \mathrm{~ms}$ later $(\Delta \sim 48$ counts $/ 10$ $\mathrm{ms}$ ). This is followed by a gradual decline (or small steps) in intensity to background level (12 s) before intensity increases again at $14.6 \mathrm{~s}$ in what appears to be a small step $(\Delta \sim 7 \pm 3$ counts $/ 10 \mathrm{~ms})$. Figure 4 (inset) is a histogram of the changes in intensity. Distinct peaks 


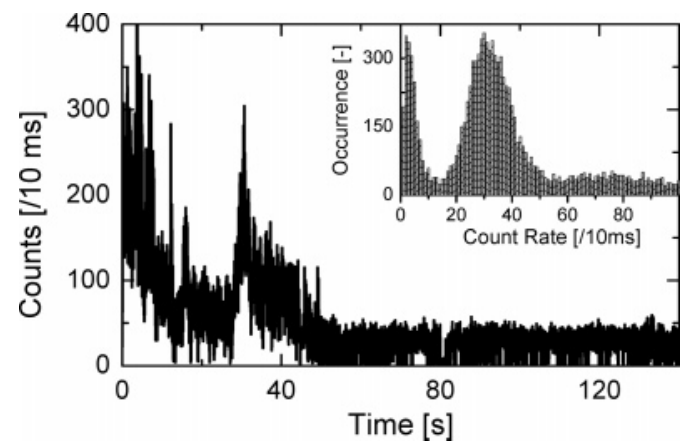

Figure 5. First $140 \mathrm{~s}$ of the fluorescence time trace (main figure) and histogram (inset) of a single long-chain DOO-PPV polymer under excitation with linearly polarized light. Bin size is $10 \mathrm{~ms}$. Excitation irradiance is $1600 \mathrm{~W} / \mathrm{cm}^{2}$. Note the peaks at 3 counts $/ 10 \mathrm{~ms}$ and 31 counts $/ 10 \mathrm{~ms}$ on the histogram.

occur at $\Delta= \pm 48$ counts $/ 10 \mathrm{~ms}$ (indicated by arrows), indicating that a section of the polymer enters and leaves a "dark state" 11 times during the polymer's survival time.

For the polymer in Figure 5, during the first $50 \mathrm{~s}$, there is a combination of large abrupt intensity jumps $(\Delta \sim 170$ counts $/ 10 \mathrm{~ms})$ and much small intensity changes on the $10 \mathrm{~ms}$ time scale of this experiment. The last of these large jumps occurs at $12.1 \mathrm{~s}$ and then drops back by the same amount $100 \mathrm{~ms}$ later. The total emission intensity gradually declines to a low $\sim 40$ counts/10 ms at $13 \mathrm{~s}$ and then fluctuates gradually until about $44 \mathrm{~s}$, reaching a maximum of $\sim 250$ counts $/ 10 \mathrm{~ms}$ at $31 \mathrm{~s}$. Although during this interval, the fluctuations in intensity are generally gradual, there are a number of discrete intensity changes of $\Delta \sim 28$ counts $/ 10 \mathrm{~ms}$. After $50 \mathrm{~s}$ there are no longer gradual changes in emission, but rather the fluorescence time trace is similar to the single step "on/off" blinking seen in small molecules. The histogram (Figure 5, inset) shows that the change in intensity is $\Delta \sim 28$ counts $/ 10 \mathrm{~ms}$ in this region.

From Figures 3-5, it can be seen that the fluorescent time trace of a polymer is characterized by abrupt quantized changes in intensity superimposed on gradual changes in emission intensity (changes $<$ shot noise). The relative size of these large jumps varies, even within a single processing batch, from individual to individual and ranges from zero to almost $100 \%$ of the total intensity. Within a single polymer, the size of the abrupt change in intensity takes on only a few distinct values (in Figure $3, \Delta \sim 19 \pm 5$ counts/10 ms; in Figure $4, \Delta \sim 48$ counts $/ 10 \mathrm{~ms}$; in Figure 5 , either $\Delta \sim 170$ or $\Delta \sim 28$ counts/10 ms.) This behavior is quite different from the single step "on/off" blinking behavior in small molecules. ${ }^{2,30}$ Rather, it seems to be a combination of the two different types of emission reported from $\mathrm{MEH}-$ PPV(discrete levels and jumps ${ }^{8,9,31}$ and exponential decaying without discrete intensity jumps ${ }^{9}$ ). The combination of a few large abrupt changes in intensity and many smaller changes suggests that the polymer is composed of two types of regions-those responsible for the large jumps in intensity and those responsible for the more gradual changes. In the first region threedimensional energy transfer efficiently couples energy to one or two emission sites (energy funnel) from a large number of absorption sections. ${ }^{8}$ In the second region, many sections both absorb and emit. (This may be due of relatively inefficient one-dimensional energy transfer along the polymer backbone. ${ }^{6,32,33}$ ) For the rest of this

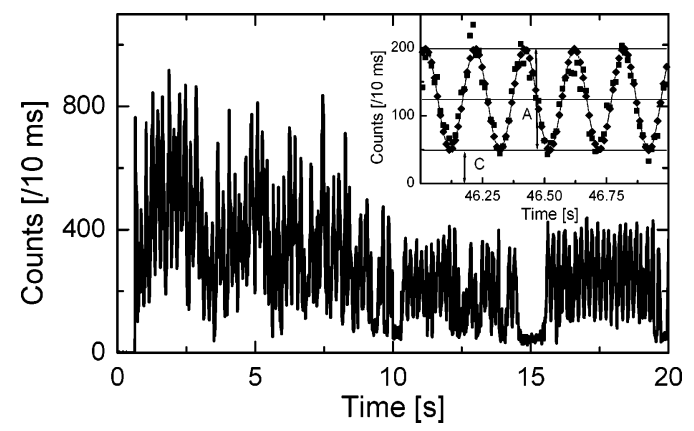

Figure 6. Fluorescent transient of a single DOO-PPV molecule under excitation with slowly rotating linearly polarized light. Excitation irradiance is $1600 \mathrm{~W} / \mathrm{cm}^{2}$. Upper inset shows an example of curve fitting to the equation $I=A \sin ^{2}(\omega t+\varphi)$ $+C$ (see text for details).

paper we will denote the first type of emission as core emission and the second as emission coming from the extended region of the polymer.

Time-Averaged Modulation Depth. Information on the overall alignment of these absorbing sections (dipoles) is obtained by rotating the polarization of the excitation light and observing the change in the total unpolarized emission intensity. This is illustrated in the following example. The fluorescent transient of a single molecule in which the excitation light is rotated is shown in Figure 6. The inset shows the result of fitting a small section of the experimental data by using $I=A$ $\sin ^{2}(\omega t+\varphi)+C$, where $I$ is the unpolarized fluorescence intensity, $\omega$ is twice the frequency of rotation of the excitation polarization $(2.5 \mathrm{~Hz}), \varphi$ is a phase factor, $C$ is the minimum emission intensity, and $A$ is the amplitude of the modulation. $A, \varphi$, and $C$ are allowed to vary to obtain the best least-squares fit. The modulation depth $(M)^{7}$ then be calculated: $M=A /(A+C) . M$ ranges from 0 (no modulation, $A=0)$ to $1(100 \%$ modulation, $C=0$ ). (A feel for the significance of $M$ can be gained by considering a polymer having only two incoherent absorption dipoles. If the dipoles are aligned, $M=1 . M=0$ indicates a $90^{\circ}$ difference in orientation, and $M=0.7$ indicates a $45^{\circ}$ misalignment.) For a straight polymer chain $M=1$, as all the absorption dipoles are aligned. In the opposite extreme, a value of $M=0$ indicates a conformation in which the absorption dipoles are have no preferred orientation. The polymer illustrated in Figure 6 was characterized by $M_{\text {average }}=$ 0.75 , indicating considerable alignment of the absorption dipoles within the polymer as a whole.

The average modulation depth, over the lifetime of the polymer, was calculated for 79 individual polymers. Figure 7 summarizes the results. The average modulation depth of $\sim 0.50$ and the almost complete absence of modulation depths $<0.3$ or $>0.7$ indicates that dipoles are neither perfectly aligned nor completely isotropically arranged. The lack of deep modulation indicates that, in contrast to short-chain polymers, long-chain polymers do not collapse into ordered rodlike structures. The lack of modulation depths $<0.3$ indicates that polymers adopt neither a highly ordered structure such as a toroid nor a highly disordered structure such as a random coil or molten globule, for which one expects $M=0$. The width $(\mathrm{fwhm}=0.31)$ indicates that there is substantial conformational variation between polymers. One possible structure satisfying these requirements would be one that combines regions of rodlike polymer with a molten globule-like region. 


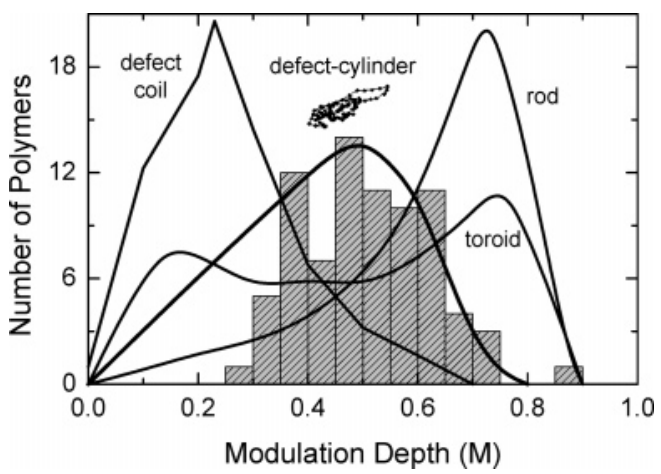

Figure 7. Distribution (HM) of the modulation depths $(M)$ from single-molecule polarization spectroscopy for over 79 single DOO-PPV polymers. The value of $M$ plotted is averaged over the lifetime of the each individual polymer. For comparison, the distribution of $M$ values expected for various classes of polymer conformations predicted by simulation are reproduced from ref 7 and displayed as solid lines.

Some additional insight into the overall polymer conformation can be obtained by comparing the histogram of observed modulation depth with that predicted for Hu's ${ }^{7}$ six classes of polymer conformation obtained under the assumption that the predominately cylindrical polymer can take any three-dimensional orientation. The model predictions are superimposed on Figure 7. For MEH-PPV, Hu et al. found that polymers adopted a cylindrical conformation and their experimental histogram was a superposition equal portions of the defectcoil and defect-cylinder structures. ${ }^{7}$ The key difference between the two structures is that the first is derived assuming that there is no Leonard-Jones attraction between sections of the polymer chain. The results of Hu's modeling cannot be compared with our experimental results quantitatively as their simulation assumes individual polymers can be randomly oriented in three dimensions while our experimental setup can best be described as two-dimensional (i.e., DOO-PPV polymers lie predominately in the sample plane). The twodimensional experimental results can be compared qualitatively to theory by noting that the effect of allowing a cylindrical structure to take an arbitrary $3 \mathrm{D}$ position is to broaden the $2 \mathrm{D}$ distribution and reduce the mean value of $M$. For instance, for completely aligned dipoles (i.e., rod-shaped) the mean $M$ is reduced from $\sim 1$ to $\sim 0.7$ with the extra degree of freedom. With this proviso, the histogram for DOO-PPV is clearly neither rod-shaped nor toroidal but rather resembles that for a class of polymer shapes denoted by $\mathrm{Hu}^{7}$ as defect-cylinders. It is interesting that DOO-PPV, despite having a very different chemical structure from $\mathrm{MEH}$ PPV, does not differ more in conformation.

Time-Dependent Modulation Depth. Observing the time dependence (or intensity dependence) of the modulation depth provides information on the relative orientation of the absorbing sections within the polymer. While the majority of polymers have both gradual and abrupt intensity changes, Figures 8 and 9 compare the time behavior of modulation depth recorded for a polymer which lacks large abrupt changes in intensity (Figure 8) with a polymer in which such abrupt changes dominate (Figure 9). For the polymer in Figure 8, the average modulation depth is $\sim 0.63$. $M$ varies only slightly over the life of the polymer and is uncorrelated with the emission intensity despite intensity changing by an order of magnitude during the section of the time trace shown here. For the polymer shown in Figure 9,

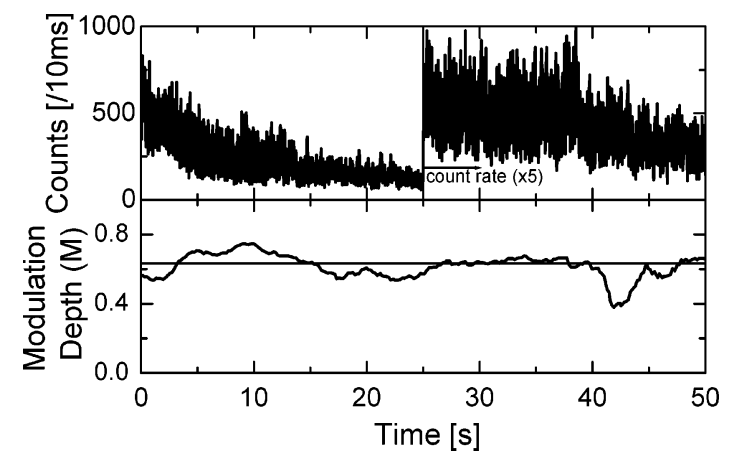

Figure 8. (top) First $50 \mathrm{~s}$ of the fluorescence time trace and (bottom) variation of the modulation depth $(M)$ over time of a single DOO-PPV polymer as obtained from single-molecule polarization spectroscopy. The horizontal line is the root-meansquare of $M$ of this polymer $(M=0.633)$. Excitation irradiance is $\sim 1600 \mathrm{~W} / \mathrm{cm}^{2}$. For this polymer, whose time decay is predominately exponential, $M$ remains approximately constant as a function of time.

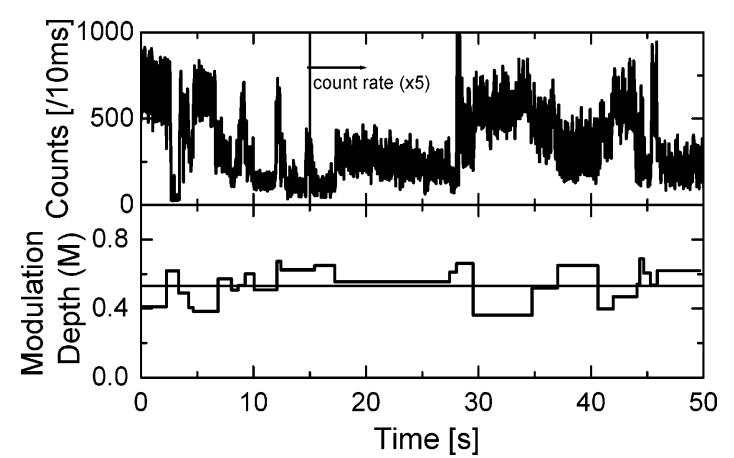

Figure 9. First $50 \mathrm{~s}$ of the (top) fluorescence time trace and (bottom) variation of the modulation depth $(M)$ over time of a single DOO-PPV polymer as obtained from single-molecule polarization spectroscopy. The horizontal line is the root-meansquare of $M$ of this polymer $(M=0.53)$. Excitation irradiance is $\sim 1600 \mathrm{~W} / \mathrm{cm}^{2}$. For this polymer, whose time decay is dominated by abrupt intensity fluctuations, $M$ changes drastically during its lifetime.

the depth of modulation is less (0.53), and $M$ varies significantly as a function of time. In addition, $M$ and emission intensity are correlated with shallower modulation at higher intensities. This fact that modulation is deeper for the polymer lacking quantized large intensity changes (Figure 8) suggests that there is greater anisotropy in the orientation of absorption dipoles in the region responsible for gradual changes.

This can be confirmed by observing the change in $M$ as the region responsible for abrupt intensity changes is alternatively quenched and unquenched. If the alignment of absorption dipoles in the core region is relatively anisotropic compared to the extended portion of the polymer, then a sudden drop in intensity when emission ceases from the core region should be correlated with an increase in modulation depth as the more anisotropically aligned outlying regions provide a greater percentage of PL. When the core region starts emitting again, there should be a corresponding decrease in the modulation depth, as is seen in Figure 9. In Figure 10 a histogram of the ratio of depth of modulation $\left(M_{\mathrm{r}}=\right.$ $\left.M_{\text {low intensity }} / M_{\text {high intensity }}\right)$ on either side of the abrupt intensity changes occurring in 38 polymers is plotted. One expects this to be Gaussian with mean $\left(\left\langle M_{\mathrm{r}}\right\rangle\right)$ dependent on the relative isotropy of the core and extended regions (i.e., if core regions are more isotropic (anisotropic) than the extended regions, then one ex- 




Figure 10. Histogram of the ratio of depth of modulation $\left(M_{\text {low intensity }} / M_{\text {high intensity }}\right)$ on either side of abrupt intensity changes for 38 single polymers. Superimposed on the experimental data are the fits to a single (dotted gray line) and a double peaked Gaussian (solid line) [see text for details].

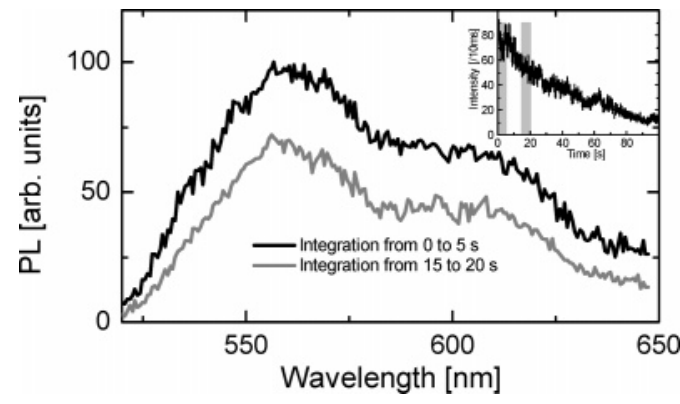

Figure 11. Spectrum at two time intervals for a polymer whose decay exhibits predominately exponential behavior. The inset shows the corresponding fluorescence time trace. Despite the intensity dropping 35\% between the first and second time interval, there is very little change in the PL spectrum.

pects $\left.M_{\mathrm{r}}>1\left(M_{\mathrm{r}}<1\right)\right)$. The best fit to a single Gaussian is centered at $M_{\mathrm{r}}=1.05$ (Figure 10, gray dotted line). $M_{\mathrm{r}}>1$ indicates that the core region is, in general, characterized by a more isotropic distribution of absorption chromophores than the extended regions. However, the histogram itself is decidedly non-Gaussian as evidenced by the high value of $\chi^{2}$ (2.6). A much better fit $\left(\chi^{2}=1.5\right)$ is obtained when one assumes that the histogram is composed of two components (Figure 10, black solid line). The large component has a peak centered at $M_{\mathrm{r}}=1.07$ with weight of 4.0 and a small component with peak centered at $M_{\mathrm{r}}=0.97$ and weight of 0.3 . This suggests that while in $93 \%$ of polymers there is a more isotropic arrangement of absorption dipoles in the "core" region than in the "extended" region, in a significant minority $(7 \%)$ of polymers, the extended region has a slightly more isotropic arrangement of dipoles.

Time-Dependent Spectra. Further insight into the two types of regions can be obtained from the variation of the spectra during the decay of the PL time trace. Figure 11 shows the spectrum of a polymer at full intensity and after the intensity has dropped to $65 \%$ of the original intensity. The time decay of the PL of this polymer is gradual with only relatively small abrupt fluctuations in intensity (Figure 11, inset). The spectra are peaked at $\sim 560 \mathrm{~nm}$ with a shoulder at $\sim 600 \mathrm{~nm}$ having a relative height of 0.67 . The spectrum exhibits no drastic change between during the decay although the peak position may shift slightly. Such a gradual spectral change is indicative of a large number of emitters taking part in the emission process. Figure 12 shows the spectra of another polymer at two periods in its time trace. In contrast to the previous polymer, the

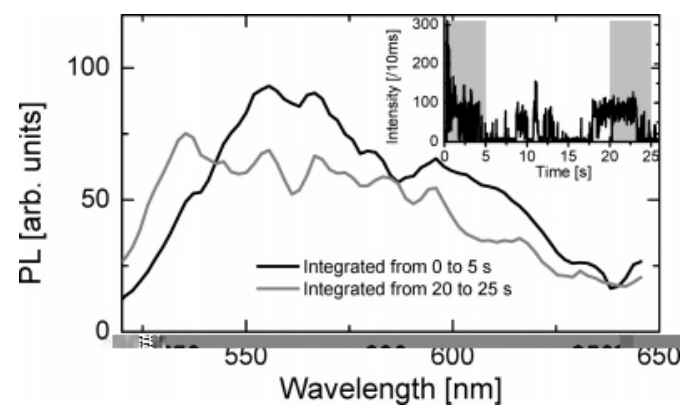

Figure 12. Spectrum at two time intervals for a polymer whose decay is dominated by abrupt changes in intensity. The inset shows the corresponding fluorescence time trace. For this polymer emission is primarily at two intensity levels. Both the spectrum peak and the relative intensity of the first and second peaks change.

time decay of the PL of this polymer is composed of large abrupt fluctuations and a noticeable absence of gradual changes in intensity (Figure 12, inset). Initially, when more than one segment is emitting, the spectrum is peaked at $\sim 560 \mathrm{~nm}$ with a shoulder at $\sim 600 \mathrm{~nm}$ having a relative height of $\sim 0.72$. However, in contrast to the stable spectrum in Figure 11, the large abrupt change in intensity between the first and second time interval is accompanied by an equally abrupt spectral change with the peak shifting $25 \mathrm{~nm}$ to $535 \mathrm{~nm}$ and the ratio of shoulder to main peak reaching close to 1 . Such drastic spectral change could only occur if there are only a few emitters in this polymer. Reiterating, the relatively constant spectra during gradual decay points to the existence of multiple emitters, while the abrupt spectral changes accompanying an abrupt intensity change points to the existence of a few emitters.

Summarizing, the above experimental results indicate the coexistence of an extended and a core region(s) within a single DOO-PPV polymer. In the core region, a relatively isotropic distribution of absorption dipoles funnels energy efficiently to one (or a few) emission sites. In the extended region, composed of a relatively anisotropic distribution of absorption dipoles, greater separation of adjacent chains forces energy transfer to occur primarily along the polymer backbone (intrachain), thus allowing for a large number of emission sites. For DOO-PPV, within the same polymer batch, the number of segments falling within each of these regions varies from individual to individual, ranging from 0 to $100 \%$, with the majority of individuals having segments in each region. For MEH-PPV, a number of groups ${ }^{11,12,32}$ have published fluorescence time traces. These can be roughly divided into two categories - those demonstrating smooth exponential decay like features and those demonstrating periods of constant emission punctuated by abrupt intensity changes. The nature of the trace observed depends on deposition conditions. In contrast, both features are often observed within a single DOO-PPV polymer and a variety of behaviors seen within a single batch. This may be a consequence of the limited solubility of this polymer that gives it a greater tendency to self-aggregate.

Rather than monitoring the orientation of absorption dipoles, Huser ${ }^{9,10}$ investigated the conformation of single MEH-PPV by observing the polarization of the emitters. For MEH-PPV dissolved in chloroform (extended, many emitters) polarization was isotropic while that dissolved in toluene ("aggregated", few emitters) exhibited high polarization anisotropy. They concluded extended MEH- 
PPV assumes a random extended coil, while the emission dipoles in aggregated MEH-PPV are aligned. This is clearly in contrast to our absorption measurements in DOO-PPV in which the reverse behavior was seen. While for the core region a consistent picture for both MEH-PPV and DOO-PPV can be obtained in which randomly aligned absorbing dipoles (i.e., molten globule) transfer energy to a few aligned emitting segments, the same is not true for the extended region. In this case, it would seem that DOO-PPV tends to extend linearly while MEH-PPV tends to loosely coil. Further experiments monitoring the conformation of the absorbing segments of MEH-PPV would be necessary to confirm this picture.

If in this experiment we are not looking at single polymers but rather groups of polymers or even a multipolymer aggregate, ${ }^{29}$ a number of alternative explanations of our data are possible. As the usual quantum jump on/off signature of single small molecules is not necessarily applicable to long chain polymers, we propose five criteria to establish that one is indeed looking at single polymers. (1) The source of the fluorescence should be diffraction limited. In this experiment, we observed fwhm $<0.4 \mu \mathrm{m}$ for isolated emission centers-close to the diffraction limit of emitted light. (2) A variation of polymer concentration should result in a corresponding variation in the density of diffraction-limited spots. The concentration of the polymer in the solvent was varied by a factor of 10 , and a corresponding variation was seen in the number of spots observed in a $100 \mu \mathrm{m}^{2}$ scan. (3) The total fluorescence intensity from a spot should not exceed the number of photons expected from a single polymer. For short chain DOO-PPV polymers prepared under similar conditions, $\sim 4000 \mathrm{cps}$ are detected. ${ }^{3}$ Since these polymers are $\sim 120$ times heavier, given equal quantum efficiency, the count rate should not exceed $500000 \mathrm{cps}$ for a single polymer. Our maximum count rates are a factor of 4 lower. (4) A PL spectrum similar to that of the polymer in dilute solution rather than that of a multipolymer aggregate ${ }^{29}$ is indicative of a single polymer (although a spectrum similar to an aggregate does not exclude the possibility that the source is a single polymer). The recorded spectrum (Figure 2) shows no evidence of large-scale multipolymer aggregation. (5) A modulation of PL intensity when the polarization of the excitation light is rotated should be indicative of a single polymer. While, a priori, one cannot predict the PL dependence on excitation polarization of a single polymer, in a large cluster one expects there to be little dependence. In the observed polymers, modulation depth was $>0.5$, indicating a significant dependence of PL intensity on excitation polarization. While any one of these five criteria does not ensure that one is looking at a single molecule, the fact that all the criteria are met gives us confidence that we are actually observing single polymer photophysics.

Finally, we would like to make some comments on the different number of photons collected in short- and long-chain polymers. Under these experimental conditions, we collect, on average, for polymers containing four excitons $\sim 1000$ [photons/s/oligomer], while for these long chain polymers containing 500 excitons, we only collect $\sim 200$ [photons/s/oligomer]. This could be either due to the statistically higher probability to create quench sites in a longer polymer or because excitons in the core region do not contribute efficiently to emission, as has been observed for aggregated states in solution ${ }^{19}$. This reduced yield could be due to a single emitter being cycled near its maximum rate. On the basis of an excited-state lifetime of $\sim 450 \mathrm{ps},{ }^{23}$ intersystem crossing time $\left(\tau_{\text {isc }}\right)$ of $\sim 40 \mathrm{~ns}$, and a triplet state lifetime $\left(\tau_{\text {triplet }}\right)$ of $\sim 20 \mu \mathrm{s}$, one expects a maximum cycling rate for an exciton of $\sim 1 \mathrm{MHz}$. In short chains of DOO-PPV (no energy transfer), at our excitation levels and assuming a fluorescence quantum yield of $0.3^{23}$ and a collection efficiency of $\sim 3 \%$, a single oligomer is cycling at $<10 \%$ of saturation. If, however, in the aggregated region 50 absorbing dipoles transmit energy to a single emitter, then clearly a bottleneck would arise, greatly limiting the total emission from the aggregated region. This is consistent with our observation that, in general, the fluorescence intensity is higher for individual polymers lacking large abrupt intensity changes. It is also consistent with the small jump of $\sim 700$ [photons/s] clearly visible just before $15 \mathrm{~s}$ in Figure 4, being interpreted as the signal from a single oligomer exiting a dark state in the extended region; i.e., emission in extended region is unchanged. (Since such a small step is indistinguishable from shot noise when there are more then 10 chromophores emitting, changes in intensity due to individual excitons turning on and off in the extended region are only distinguishable when the majority of the chromophores have already been quenched, i.e., the tail.)

\section{Conclusion}

In conclusion, the fluorescence time trace of a single DOO-PPV is a combination of a few large quantized jumps along with gradual changes in intensity. The relative contribution to the total intensity of the two behaviors varies from individual to individual within the same processing batch. Time-independent polarization spectroscopy indicates considerable alignment of absorption dipoles within a polymer demonstrating that the polymer is neither rod-shaped, toroidal, or a molten globule. Time-dependent polarization data indicate that the distribution of absorption dipoles in the region of polymer responsible for large abrupt changes in intensity is more isotropic then the region responsible for more gradual changes in intensity. These results suggest the coexistence of an extended and a core region within a single polymer. The extended (intrachain) region is characterized by a strongly anisotropic distribution of absorption dipoles and multiple emitters. Absorption dipoles in the core region, while still exhibiting alignment, are relatively more isotropically distributed. In this region, close packing allows efficient threedimensional energy transfer to funnel energy from many absorbing to a few emitting sites. For DOO-PPV, under similar processing conditions and within the same batch, the relative size of the core and extended regions varies from individual to individual. While it is tempting to try to equate the extended region observed in this single polymer experiment with the intrachain state and the core region with the aggregate state observed in ensemble experiments, ${ }^{19}$ our spectral data are insufficient to make this connection.

Acknowledgment. This research is supported by National Science Council and MOE Program for Promoting Academic Excellence of Universities under Grant 91-E-FA04-4A. 


\section{References and Notes}

(1) Friend, R. H.; Gymer, R. W.; Holmes, A. B.; Burroughes, J. H.; Marks, R. N.; Taliani, C.; Bradley, D. D. C.; Dos Santos, D. A.; Bredas, J. L.; Logdlund, M.; Salaneck, W. R. Nature (London) 1999, 397, 121.

(2) Betzig, E.; Chichester, R. J. Science 1993, 262, 1422.

(3) White, J. D.; Hsu, J. H.; Yang, S. C.; Fann, W. S.; Pern, G. Y.; Chen, S. A. J. Chem. Phys. 2001, 114, 3848.

(4) Yan, M.; Rothberg, L. J.; Papadimitrakopoulos, F.; Galvin, M. E.; Miller, T. M. Phys. Rev. Lett. 1994, 73, 744.

(5) White, J. D.; Hsu, J. H.; Fann, W. S.; Yang, S. C.; Pern, G. Y.; Chen, S. A. Chem. Phys. Lett. 2001, 338, 263.

(6) Wang, C. F.; White, J. D.; Lim, T. L.; Hsu, J. H.; Yang, S. C.; Fann, W. S.; Peng, K. Y.; Chen, S. A. Phys. Rev. B 2003, 67, 035202

(7) Hu, D.; Yu, J.; Wong, K.; Bagchi, B.; Rossky, P. J.; Barbara, P. F. Nature (London) 2000, 405, 1030.

(8) Yu, J.; Hu, D.; Barbara, P. F. Science 2000, 289, 1327.

(9) Huser, T.; Yan, M. J. Photochem. Photobiol. A: Chem. 2001, $144,43$.

(10) Huser, T.; Yan, M.; Rothberg, L. J. Proc. Natl. Acad. Sci. U.S.A. 2000, 97, 11187.

(11) Kumar, P.; Mehta, A.; Dadmun, M. D.; Zheng, J.; Peyser, L.; Bartko, A. P.; Dickson, R. M.; Thundat, T.; Sumpter, B. G.; Noid, D. W.; Barnes, M. D. J. Phys. Chem. B 2003, 107, 6252.

(12) Sartori, S. S.; De Feyter, S.; Hofkens, J.; Van der Auweraer, M.; De Schryver, F.; Brunner, K.; Hofstraat, J. W. Macromolecules 2003, 36, 500 .

(13) Frolov, S. V.; Gellermann, W.; Ozaki, M.; Yoshino, K.; Vardeny, Z. V. Phys. Rev. Lett. 1997, 78, 729.

(14) Frolov, S. V.; Vardeny, Z. V.; Yoshino, K. Phys. Rev. B 1998 $57,9141$.

(15) Frolov, S. V.; Bao, Z.; Wohlgennannt, M.; Vardeny, Z. V. Phys. Rev. Lett. 2000, 85, 2196.

(16) Frolov, S. V.; Lane, P. A.; Gellermann, W.; Vardeny, Z. V. Phys. Rev. Lett. 1997, 78, 4285.

(17) Hsu, J. H.; Fann, W. S.; Tsao, P. H.; Chen, K. R. Chuang and, S. A. J. Phys. Chem. A 1999, 103, 2375.

(18) Chang, R.; Hsu, J. H.; Fann, W. S.; Liang, K. K.; Chang, C. H.; Hayashi, M.; Yu, J.; Lin, S. H.; Chang, E. C.; Chuang, K.
R.; Chen, S. A. Chem. Phys. Lett. 2000, 317, 142.

(19) Chang, R.; Hsu, J. H.; Fann, W. S.; Yu, J.; Lin, S. H.; Lee, Y. Z.; Chen, S. A. Chem. Phys. Lett. 2000, 317, 153.

(20) Becker, H.; Spreitzer, H.; Ibrom, K.; Kreuder, W. Macromolecules 1999, 32, 4925 .

(21) Sarneck, G. L.; L Burn, P.; Kraft, A.; Friend, R. H.; Holmes, A. B. Synth. Met. 1993, 55-57, 914.

(22) Askari, S. H.; Rughoophth, S. D.; Wudl, F. Synth. Met. 1989, 29, E129.

(23) Hsu, J. H.; Hayashi, M. T.; Lin, S. H.; Fann, W. S.; Rothberg, L. J.; Perng, G. Y.; Chen, S. A. J. Phys. Chem. B 2002, 106, 8582.

(24) Sun, W. Y.; Hsu, J. H.; Yang, S. C.; White, J. D.; Fann, W. S. J. Lumin. 2002, 98, 41.

(25) White, J. D.; Sun, W. Y.; Su, S. C.; Hsu, J. H.; Lim, T. L.; Fann, W. S. J. Lumin. 2002, 98, 45

(26) White, J. D.; Hsu, J. H.; Wang, C. F.; Chen, Y. C.; Hsiang, J. C.; Su, S. C.; Sun, W. Y.; Fann, W. S. J. Chin. Chem. Soc. 2002, 49, 669

(27) Ha, T.; Enderle, Th.; Chemla, D. S.; Weiss, P. R.; Selvin, S. Phys. Rev. Lett. 1996, 77, 3979.

(28) Fourkas, J. T. Opt. Lett. 2001, 26, 211.

(29) Scheblykin, I.; Zoriniants, G.; Hofkens, J.; De Feyter, S.; Van der Auweraer, M.; De Schryver, F. C. ChemPhysChem 2003, 4, 260.

(30) English, D. S.; Furube, A.; Barbara, P. F. Chem. Phys. Lett. 2000, 324, 15

(31) Hu, D.; Yu, J.; Barbara, P. F. J. Am. Chem. Soc. 1999, 121, 6936.

(32) Nguyen, T. Q.; Wu, J.; Doan, V.; Schwartz, B. J. Science 2000, 288,652

(33) Nguyen, T. Q.; Wu, J.; Tolbert, S. H.; Schwartz, B. J. Adv. Mater. 2001, 13, 609.

(34) Kohler, B. E.; Samuel, I. D. W. J. Chem. Phys. 1995, 103, 6248. Kohler, B. E.; Woehl, J. C. J. Chem. Phys. 1995, 103, 6253.

MA048686T 